\title{
CINÉTICA DA ABSORÇÃO DO NITROGENIO DURANTE BORBULHAMENTO EM FORNO PANELA*
}

\author{
Bruno Veiga Fontana ${ }^{1}$ \\ Andre Luiz Vasconcellos da Costa e Silva ${ }^{2}$ \\ Pedro Henrique Coutinho ${ }^{3}$ \\ Vanderley Ramos Lamim ${ }^{4}$
}

\section{Resumo}

O uso do nitrogênio como gás de injeção através de plug poroso no forno panela pode ser importante do ponto de vista econômico, no entanto pode provocar pickup excessivo de nitrogênio caso seja realizado de forma inadequada. No presente trabalho, são apresentados os resultados de testes de injeção de nitrogênio e nitrogênio seguido de argônio em aços para produtos longos. Visando compreender os processos de absorção e sua cinética, um modelo cinético é formulado e ajustado aos dados experimentais. $O$ modelo cinético clássico descreve bem os resultados experimentais e permite o uso para previsões do efeito da injeção de nitrogênio sobre o teor de nitrogênio no aço. Observa-se que a cinética de absorção é controlada pela agitação da panela e pela presença de elementos tenso-ativos, notadamente o oxigênio, como esperado, de acordo com os modelos clássicos do fenômeno. A técnica da injeção de nitrogênio seguida de argônio é bem-sucedida economicamente e o modelo permite a previsão e o controle da absorção de nitrogênio pelo aço.

Palavras-chave: Cinética; Nitrogênio; Forno panela; Aço; Modelamento.

\section{KINETICS OF NITROGEN ABSORPTION DURING NITROGEN INJECTION IN THE LADLE FURNACE}

\section{Abstract}

The use of nitrogen as agitation gas injected through the ladle porous plug can result in significant economic savings. However, it can result in excessive nitrogen pick-up if performed in an uncontrolled way. In this work, the results of tests of nitrogen injection followed by argon injection in steel long product processing are presented. In order to understand the kinetics of the process we attempted to adjust a simple kinetic model to the data. This simple model describes well the experimental data and is useful in determining the effects of the injection time on the steel nitrogen content. The absorption kinetics is controlled by the ladle agitation and by the presence of surface active elements, in special oxygen and sulfur, as expected, based on the classical models that describe the phenomenon. The technique of nitrogen injection followed by argon is successful from the economical point of view and the initial simple model formulated in this work makes it possible to predict and control nitrogen absorption by the liquid steel.

Keywords: Kinetics; Ladle furnace; Steel; Modeling; Nitrogen.

Engenheiro Metalúrgico, Votorantim Resende, RJ, Brasil.

Professor, PhD, EEIMVR-UFF, Volta Redonda, RJ, Brasil.

Engenheiro Metalúrgico, Votorantim Resende, RJ, Brasil.

Técnico de Processos, Votorantim Resende, RJ, Brasil. 


\section{INTRODUÇÃO}

A agitação do aço no tratamento em metalurgia de panela é essencial para a dissolução e homogeneização das ligas adicionadas, da composição química e da temperatura assim como para o progresso das reações desejadas (desoxidação e dessulfuração)[1,2]. Os dois métodos mais usuais de agitação são a agitação eletromagnética e a injeção de gás inerte por tijolos porosos, sendo o segundo muito mais usual, atualmente $[3,4]$.

A escolha tradicional, na agitação com gás inerte, recai sobre o argônio, pela sua baixíssima solubilidade no aço líquido. Entretanto, o custo do argônio é significativo e diversas outras opções já foram consideradas, tais como $\mathrm{CO}_{2} \mathrm{e}$ $\mathrm{N}_{2}$. O uso de CO2 conduz, normalmente, a oxidação indesejada. Como a absorção do nitrogênio pelo aço líquido é bastante sensível a presença de residuais tenso-ativos, como oxigênio e enxofre, principalmente [5,6], o uso deste gas, se realizado com os cuidados necessários, pode ser uma opção interessante em função da redução de custos e, até, da incorporação (pick-up) controlado deste intersticial. Assim, uma prática de uso de gases em sequência, como, por exemplo, nos conversores de sopro combinado [7], em que nitrogênio é usado por um tempo definido, em função de um limite máximo deste intersticial aceitável no aço, seguido pela troca por argônio, é uma opção bastante interessante, economicamente, para uma série de produtos siderúrgicos.

No presente trabalho são revistas as características do processo de absorção e remoção do nitrogênio do aço e os modelos cinéticos aplicáveis. Usando resultados experimentais é estabelecido um modelo cinético preliminar que permite definir o tempo máximo de injeção de nitrogênio e o momento da mudança da injeção para argônio. São indicadas as principais melhorias desejadas do modelo e as medidas em curso para seu aprimoramento.

\section{CINÉTICA DA ABSORÇÂO E DESORÇÃO DO NITROGENIO NO AÇO LÍQUIDO}

O nitrogênio se dissolve no aço de forma atômica seguindo a lei de Sievert [2]. O limite de solubilidade do nitrogênio, a 1 atm, no ferro é mostrado na Figura 1

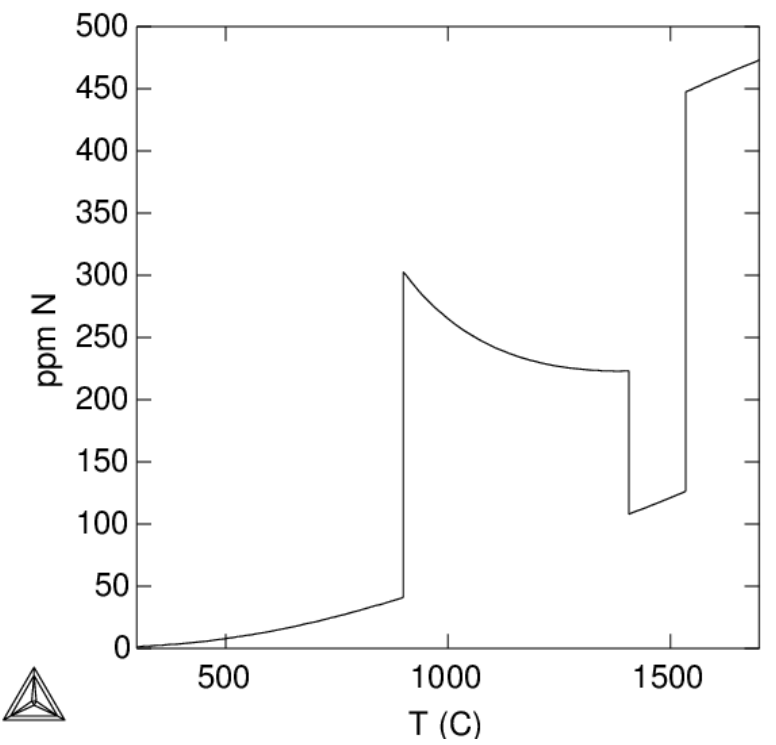

Figura 1. Solubilidade do nitrogênio no ferro puro a $1 \mathrm{~atm}$. Calculado [8] [9] 
Como qualquer processo de cinética heterogênea, em siderurgia, a entrada no nitrogênio no aço líquido ou sua remoção envolve as etapas de transporte do nitrogênio atômico no metal, reação química na interface $\left(2 \underline{N} \leftrightarrow N_{2}\right)$ e transporte do nitrogênio gasoso na fase gás.

Em geral, como a reação química é uma etapa suficientemente mais rápida que as etapas de transporte e, no caso de sistemas líquido-gás é mais comum se encontrar a resistência maior ao transporte na fase líquida, uma expressão do fluxo de nitrogênio absorvido pelo metal toma a forma da Equação 1:

$$
j=k\left(\% N_{e q}-\% N(t)\right)
$$

Equação 1

Onde $j$ é o fluxo, normalmente em massa por unidade de tempo e de área, $k$ é o coeficiente de transporte de massa $\% N_{\text {eq }}$ é o teor de nitrogênio em equilíbrio com o gás e $\% N(t)$ é o teor de nitrogênio no banho. Entretanto, a passagem dos átomos de nitrogênio pela interface é significativamente afetada por fenômenos de superfície como adsorção e dessorção, especialmente influenciados pela ocupação de sítios da interface metal-gás por elementos tenso-ativos [10,11,12]. Assim, os teores de oxigênio e enxofre, especialmente, tem grande influência sobre a capacidade de o aço absorver nitrogênio ou do nitrogênio ser removido do aço, sob vácuo, por exemplo.

Diversas expressões foram derivadas por vários autores para considerar o efeito dos elementos tenso-ativos na "obstrução" dos sítios da interface. Ban-ya e colaboradores [12] reviram vários modelos e sumarizaram os dois modelos mais comuns. Em ambos, assume-se que tanto o nitrogênio como o enxofre e o oxigênio ocupam os mesmos sítios interfaciais. No primeiro, desenvolvido por Ban-ya, a etapa de formação de gás a partir do nitrogênio atômico adsorvido na interface ( $2 N \leftrightarrow N_{2}+\square$ ) é considerado a etapa limitadora. No segundo modelo, desenvolvido por Darken e Turkdogan [13] e Belton [14], é considerada a ocupação da interface, limitando o número de sítios livres para a reação e a diferença de atividade (ou concentração) do nitrogênio continua sendo considerada a força motriz para o processo, de modo que o coeficiente de transporte de massa k, da Equação 1, é ajustado em função dos teores de oxigênio e enxofre em solução, que determinam a fração de sítios livres na interface, $\left(1-\theta_{O}-\theta_{S}\right)$. Este modelo é mais comumente usado e resulta em um coeficiente de transporte de massa efetivo inversamente proporcional aos teores de oxigênio e enxofre em solução. Ban-ya demonstrou a consistência dos dois modelos e determinou coeficientes, empiricamente, para ambos.

Considerando que o fluxo de nitrogênio das bolhas injetadas para o metal ocorre de acordo com a Equação 1 e que o efeito da presença dos elementos tensoativos possa ser considerado conforme o modelo de Darken, Turkdogan e Belton, é possível formular um balanço de massa de nitrogênio na panela contendo uma massa $W$ de aço e supondo que o fluxo atue sobre uma área $A$, conforme a Equação 2:

$$
\frac{W \% N(t)}{100}+j A d t=\frac{W \% N(t+d t)}{100}
$$

A integração da Equação 2, considerando o fluxo conforme a Equação 1, resulta na solução clássica dada pela Equação 3: 


$$
-\ln \left(\frac{\% N_{e q}-\% N(t)}{\% N_{e q}-N(0)}\right)=\frac{100 A k}{W} t
$$

Equação 3

Onde o coeficiente $k$ pode ser uma função dos teores de oxigênio e enxofre, como discutido acima. Embora existam modelos mais completos, em que a variação da pressão da bolha (e consequentemente de $\% N_{\text {eq }}$ ) em função da subida da bolha no interior da panela [15], optou-se por um modelo simples, para avaliação inicial do problema.

\section{MEDIDAS EXPERIMENTAIS}

Para avaliar a cinética de absorção do nitrogênio durante a injeção de nitrogênio seguida de argônio foram avaliadas um total de 4 corridas de aço ABNT 1026 elaboradas em Forno Elétrico a Arco e processadas em Forno Panela. Em todas as corridas foram medidas as temperaturas durante o processo, vazão e tempo de injeção de cada um dos gases $\left(\operatorname{Ar}\right.$ e $\left.N_{2}\right)$ e foram realizadas medições dos teores de nitrogênio no início e final do processo. Como o arco elétrico poderia produzir ionização do ar e aumentar o pick-up de nitrogênio foi medido, também, o tempo de power-on no FP. Adicionalmente, foram realizadas medidas intermediárias de teor de nitrogênio e medidas do teor de oxigênio através de sonda eletrolítica.

\section{RESULTADOS E DISCUSSÃO}

Para a aplicação do modelo cinético empregou-se a Equação 3, determinando um fator empírico único $\frac{100 A k}{W}$ através do ajuste, por mínimos quadrados, dos desvios entre os valores do termo do lado esquerdo da Equação 3, medido e calculado.

A Figura 2 apresenta o resultado do modelamento de 4 corridas em que foram realizadas medidas intermediárias do teor de nitrogênio e medidas do teor de oxigênio. Optou-se por fixar o valor do teor de nitrogênio em equilíbrio em 450ppm para evitar o ajuste de dois parâmetros. O valor escolhido é razoável para a faixa de temperatura e composição tratada no forno panela.

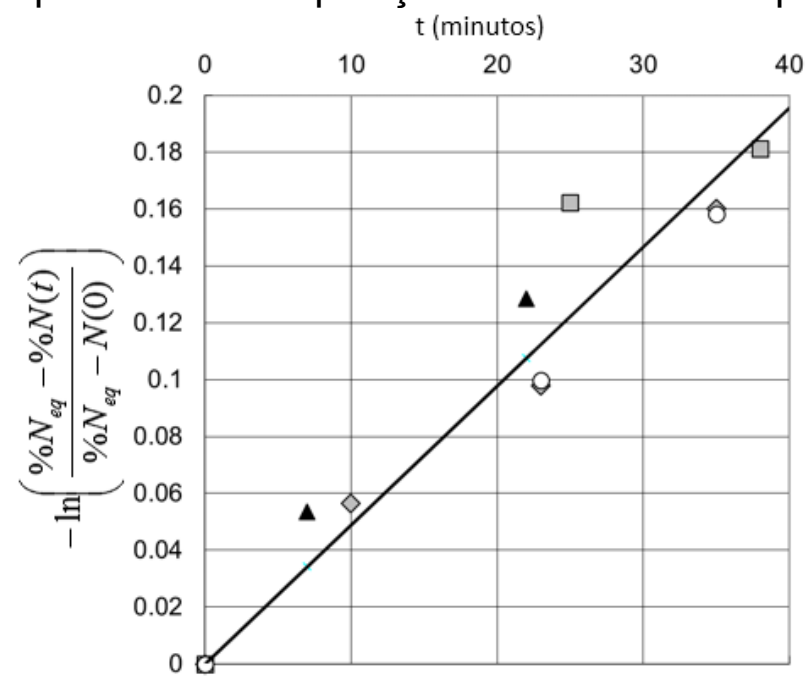

Figura 2. Ajuste da Equação 3 a quatro corridas em que houve medição intermediária do teor de nitrogênio (ver texto para discussão). 
Observa-se que o modelo se ajustou bem aos dados experimentais, tendo se obtido um valor de $\frac{100 A k}{W}=0.00489$. Com este valor, é possível empregar o modelo para prever o tempo máximo de injeção de nitrogênio para não ultrapassar determinado teor deste elemento, no aço. Assim, por exemplo, definido um limite máximo de 135ppm para o teor de nitrogênio ao fim do tratamento de uma corrida com teor inicial de $85 \mathrm{ppm}$ de nitrogênio, é possível calcular, através da Equação 4, o tempo máximo de tratamento com nitrogênio:

$$
\ln \left(\frac{450-135}{450-85}\right)=-0.00489 t
$$

Equação 4

O tempo de 30 minutos é obtido como o tempo máximo de injeção de nitrogênio, neste caso.

Além disto, para estas corridas, buscou-se observar o efeito do teor de oxigênio do banho sobre a cinética de absorção. Como o oxigênio varia durante 0 tratamento no FP, quando a desoxidação progride, é difícil definir um valor fixo para emprego no modelo. Assim não foi possível encontrar uma correlação boa empregando o modelo de Belton, por exemplo. Um modelo mais complexo, que considerasse a variação do oxigênio ao longo do processo de agitação e desoxidação, seria, talvez, mais preciso. De qualquer forma, buscou-se 3 corridas com o mesmo teor de nitrogênio inicial, tempos muito semelhantes de injeção de nitrogênio e que diferissem, apenas, pelo teor de oxigênio na chegada ao FP. A Figura 3 mostra que se observa uma correlação entre o teor de oxigênio de chegada ao FP e o pick-up de nitrogênio.

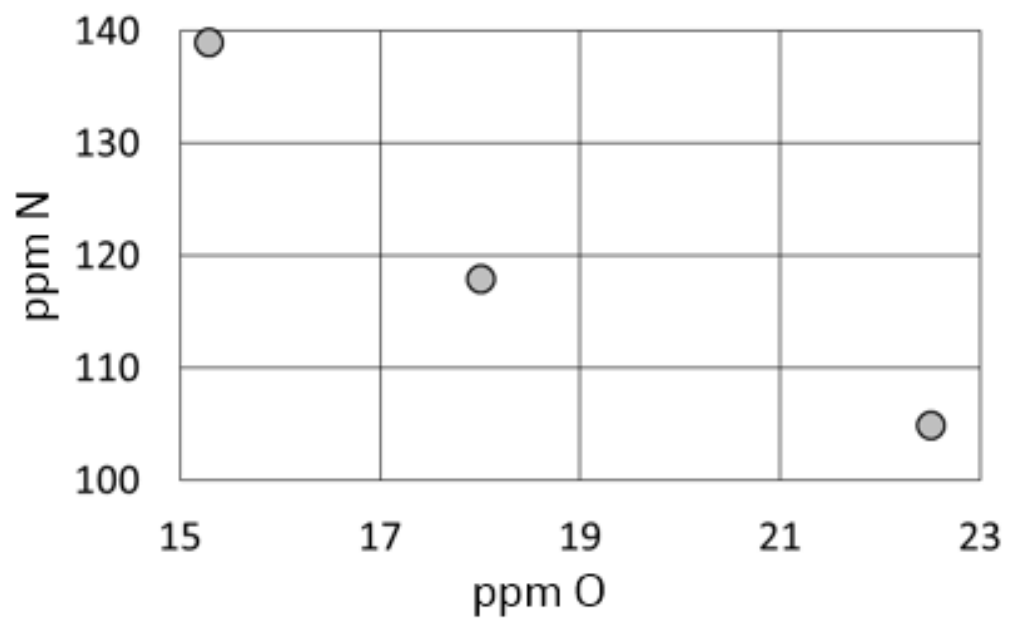

Figura 3. Teor de nitrogênio final para 3 corridas com o mesmo teor inicial de $\mathrm{N}$ e o mesmo tempo de injeção de gás $\mathrm{N}_{2}$, em função do teor de oxigênio na chegada ao FP. Ver texto para discussão.

\section{CONCLUSÕES}

Os resultados do teste de um modelo inicial foram promissores, indicando que é possível estabelecer um modelo simples capaz de auxiliar na previsão do tempo máximo de uso de injeção de nitrogênio quando um determinado limite deste elemento não deve ser excedido na corrida.

Observou-se que o teor de oxigênio dissolvido no banho tem efeito na cinética de absorção do nitrogênio, como esperado. Entretanto, como o oxigênio varia 
durante o tratamento no forno panela, não foi possível quantificar este efeito com o modelo simples inicial.

Um modelo mais complexo, que leve em conta a pressão de nitrogênio na bolha ascendente e o teor de oxigênio variável ao longo da desoxidação possivelmente fornecerá resultados mais acurados do pick-up de nitrogênio.

\section{Agradecimentos}

ACS agradece a Votorantim Siderurgia a oportunidade da realização e publicação deste trabalho.

\section{REFERÊNCIAS}

1. Bannenberg N, Bergmann B, Gaye H. Combined decrease of sulphur, nitrogen, hydrogen and total oxygen in only one secondary steelmaking operation. Steel Research. 1992;63(10):431-7.

2. Fruehan R, editor. Making, Shaping, and Treating of Steel, Steelmaking And Refining Volume. 11th Edition. Pittsburgh PA: AISE Steel Foundation; 1998.

3. Mazumdar D, Guthrie IL. The physical and mathematical modeling of gas stirred ladle systems. ISIJ International. 1995;35(1):1-20.

4. Szekely J. Ladle metallurgy. Duesseldorf: Springer Verlag; 1989.

5. Ban-Ya S, Ishii F, Iguchi $Y$, Nagasaka T. Rate of nitrogen desorption from liquid iron-carbon and iron-chromium alloys with argon. Metallurgical Transactions $B$. 1988;19(2):233-42.

6. Belton GR. How fast can we go? The status of our knowledge of the rates of gasliquid metal reactions. Metallurgical Transactions B. 1993;24B(April):241-58.

7. Kempken J, Pluschkell W. Simulationsrechnungen zur Entwicklung der Stickstoff im LD-Prozess. Stahl und Eisen. 1995;115(8):67-74.

8. Sundman B, Jansson B, Andersson J-O. Thermo-Calc, a databank for calculation of phase equilibria and phase diagrams. In: Computer calculation of phase diagrams. Orlando: ASM International, Materials Park, OH; 1986.

9. TCAB. TCFE7 Database. Stockholm: TCAB; 2012.

10. Fruehan RJ, Martonik LJ. The rate of absorption of nitrogen into liquid iron containing oxygen and sulfur. Metallurgical Transactions B. 1980;11B(December):615-21.

11. Richardson FD. The physical chemistry of melts in metallurgy. Academic Press; 1974.

12. Ban-ya S, Ishi $F$, Iguchi $Y$, Nagasaka $T$. Rate of nitrogen desorption from liquid iron-carbon and iron-chromium alloys with argon. Metallurgical Transactions B. 1988;19B(April):233-42.

13. L.S. Darken, E.T. Turkdogan. Heterogeneous Kinetics at Elevated Temperatures. In: Proceedings International Conference in Metallurgy. Plenum Press, London, England: G R Belton, W R Worrell eds.; 1970.

14. G R Belton. Langmuir Adsorption, the Gibbs Adsorption Isotherm, and Interfacial Kinetics in Liquid Metal Systems. Metallurgical Transactions B. 1976;7B:35-4.

15. Gaye $\mathrm{H}$, Huin D, Bannenberg N, Bergmann B. Modeling of vacuum tank degassing of liquid steel. Le Vide, les Couches Minces. 1992;261(Mars-Avril):55-7. 